\title{
Graves' disease complicating myelopathy
}

\author{
J H Frisbie MD, B Fisch OD
}

The Spinal Cord Injury and Optometry Services, Veterans Administration Medical Center, 940 Belmont St, Brockton 02104, MA, USA; Department of Medicine, Brigham and Womens Hospital; and New England College of Optometry, Boston, MA, USA.

Background. Exophthalmos, supraventricular arrhythmias, and myxedema have been noticed among patients chronically paralysed due to myelopathy. A survey was conducted to document and explain the observation.

Method. A long term care population of 77 male myelopathy patients aged 59 $+/-14$ years (mean $+/-1$ standard deviation), paralysed $19+/-11$ years, were compared by exophthalmometry (Hertel instrument) with 53 male non myelopathy patients and hospital employees, aged $57+/-15$ years, by review of electrocardiograms (EKGs) of record with 91 male non myelopathy hospital patients, aged $59+/-13$ years, and by thyroid function tests with standard values.

Results. Abnormal ocular protrusion $(>22 \mathrm{~mm}$ ) was found in 9 myelopathy patients $(12 \%)$ and in none of the controls. Exophthalmos was bilateral in 7 . The mean ocular protrusion was $17.2+/-3.6 \mathrm{~mm}$ for myelopathy patients and $14.9+/-3.9 \mathrm{~mm}$ for controls $(p<0.001)$. Three exophthalmic patients had atrial fibrillation. Arrhythmias had been recorded in 186 of 1390 EKGs in 34 myelopathy subjects and in 63 of 377 EKGs in 25 controls. The ratios of supraventricular to ventricular arrhythmias were 2.8 for myelopathy patients and 0.7 for controls $(p<0.02)$. Hypothroidism was found in 2 of 74 patients tested, $2.7 \%$.

Conclusion. Exophthalmos, supraventricular arrhythmias, and myxedema are unusually common among chronic myelopathy patients. Preceding Graves' disease with residual effects can be suggested as the explanation.

Key words: spinal cord injury; myelopathy; exophthalmos; arrhythmia; thyroid disease; Graves' disease.

In the course of primary care for a small population of men paralysed due to myelopathy an unexpected number of patients with residua of Graves' disease has been observed. Graves' disease is characterized mainly by 2 conditions: (a) proptosis and (b) thyrotoxicosis. ${ }^{1}$ Proptosis, once present, does not remit. ${ }^{2}$ Thyrotoxicosis, sometimes recurrent, does remit, but often with a residuum of supraventricular arrhythmia or with a natural course leading to myxedema. ${ }^{3,4}$ Hyperthyroidism was not recognized during the 5 years of observation in the population to be described, but several patients exhibited proptosis, supraventricular arrhythmia, or myxedema. Since these observations are objectively measurable, a survey was conducted to test the clinical impression of residua of Graves' disease complicating myelopathy.

\section{Methods}

\section{Subjects}

The records of 77 patients, who represented $96 \%$ of those receiving inpatient or outpatient continuing care at this spinal cord injury facility for the index year, 1986, were surveyed. All patients were male veterans, 75 were white and 2 black. The age was $59+/-14$ years (mean $+/-1$ standard deviation), range 30 to 95 ; the duration of paralysis was $19+/-11$ years, range 2 to 44 ; the median level of injury was $\mathrm{C} 6$, range $\mathrm{C} 1$ 
(incomplete) to L4 with 56 being quadriplegic. The cause of spinal paralysis was traumatic in 67 and medical in 10.

Control subjects for exophthalmometry were 53 male volunteer outpatients and hospital employees aged $57+/-15$ years, range 26 to 82 .

Control subjects for the cardiac arrhythmias were 91 male veterans receiving inpatient or outpatient care at the same institution. The age was $59+/-13$ years, range 30 to 80 . These subjects were chosen by random sampling of the electrocardiographic files stored at the hospital. None of the subjects had myelopathy.

\section{Exophthalmometry}

The Hertel instrument for exophthalmometry was used, ${ }^{5}$ and measurements were read by a single examiner (BF). The subjects were sitting upright, out of bed, for examination. ${ }^{6}$ The upper limits of normal for ocular protrusion were considered to be $22 \mathrm{~mm}$ for white men, $25 \mathrm{~mm}$ for black men, and not more than $2 \mathrm{~mm}$ for the difference between each eye. ${ }^{7}$

\section{Electrocardiography}

Routine 12 lead electrocardiography had been carried out for both groups of subjects at intervals throughout various hospitalizations and outpatient visits.

A premature atrial contraction (PAC) was recognized by a shortened $\mathrm{RR}$ interval due to a premature and deformed $P$ wave followed by an unchanged QRS complex and a normal RR interval (no compensatory pause).

A premature ventricular contraction (PVC) was designated by a shortened RR interval due to a widened and deformed QRS complex with changed initial vector and no preceding $\mathrm{P}$ wave, followed by a long $\mathrm{RR}$ interval (compensatory pause).

A junctional rhythm (JR) was designated by the criteria for premature atrial contractions except for the absence of a $\mathrm{P}$ wave or a reversal of a detectable $P$ wave vector.

Atrial tachycardia (AT) was diagnosed if the QRS complexes were regular, not de- formed, and occurring at a rate greater than 170 per minute.

Atrial flutter (AF) was designated if the $P$ rate was 250 to 350 and the QRS complexes were not widened. Atrial fibrillation (AF) was designated if a wavy baseline replaced $P$ waves, the QRS configuration was not widened, and the rhythm was irregular. ${ }^{8}$

\section{Thyroid function tests}

These tests were performed by the hospital nuclear medicine department by radioimmunoassay (Magic kit by Corning-Ciba, Medfield, MA). Thyroid function tests were recorded only if the subject had been afebrile for 30 days, to avoid the abnormalities induced by acute medical illness. ${ }^{9}$

\section{Statistical comparisons}

The myelopathy and non myelopathy exophthalmometry groups were compared for the number of subjects with abnormal readings and also by the distributions of the measurements. The significance of the difference between the means of these distributions was assessed by the Student $t$ test.

The myelopathy and non myelopathy EKG groups were subselected for subjects with electrocardiograms which showed arrhythmias. Arrhythmias were classified as supraventricular or ventricular and subjects were similarly classified according to the predominant arrhythmia recorded. The ratios of supraventricular to ventricular arrhythmias in the myelopathy and non myelopathy subjects were calculated and the difference between these ratios assessed by the chi square test.

\section{Results}

\section{Eye signs}

Nine $(12.2 \%)$ of the paralysed subjects had abnormal exophthalmometry, and 7 of these had bilaterally symmetrical exophthalmos. None of the control group had exophthalmos. The average ocular protrusion was $17.2+/-3.6 \mathrm{~mm}$, range 10 to $27 \mathrm{~mm}$ for the myelopathy patients and $14.9+/-3.9 \mathrm{~mm}$, range 8 to $22 \mathrm{~mm}$ for the controls $(\mathrm{t}=3.52$, 
$p<0.001)$. Examples of exophthalmos are shown in Figures 1 and 2.*

Extraocular movements were examined in the patients with exophthalmos and the results have been listed in Table 1 .

\section{Arrhythmia}

Thirty-four myelopathy patients had 186 arrhythmic electrocardiograms out of 1390 tracings recorded for all patients. Twentysix control patients had 63 arrhythmic EKGs out of 377 recorded for the control group. The myelopathy patients with arrhythmia showed a predominance of supraventricular arrhythmia as compared with the control group (Table 2). The likelihood of any arrhythmia being supraventricular was 4 times greater in the myelopathy patients.

\section{Thyroid function tests}

Seventy-four of the myelopathy patients had thyroid function testing.

Two patients were chemically hypothyroid with thyroid stimulating hormone (TSH) levels consistently $>20 \mathrm{uU} / \mathrm{ml}$ (normal $<5 \mathrm{uU} / \mathrm{ml}$ ) but had no complaints. After L-thyroxine adminstration, however, both patients noted easing of their usual constipation.

Two patients had repeatable elevations of

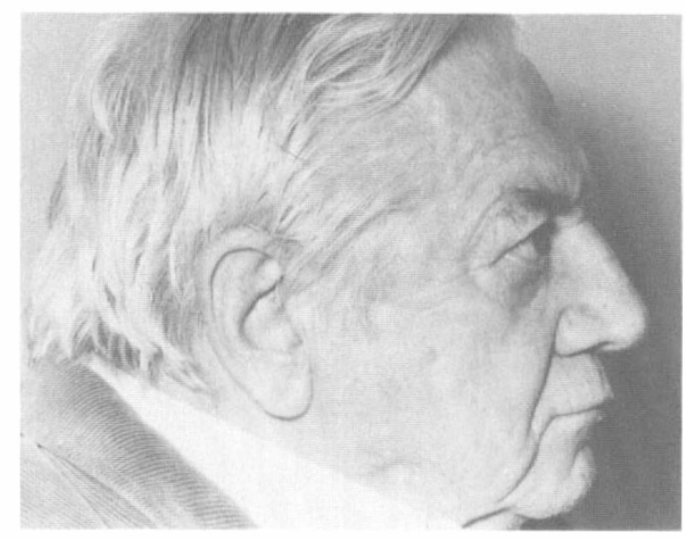

Figure 1 Lateral view of a subject with $24 \mathrm{~mm}$ exophthalmos.

\footnotetext{
*Written signed permission for the publication of Figures 1 and 2 was obtained by the authors.
}

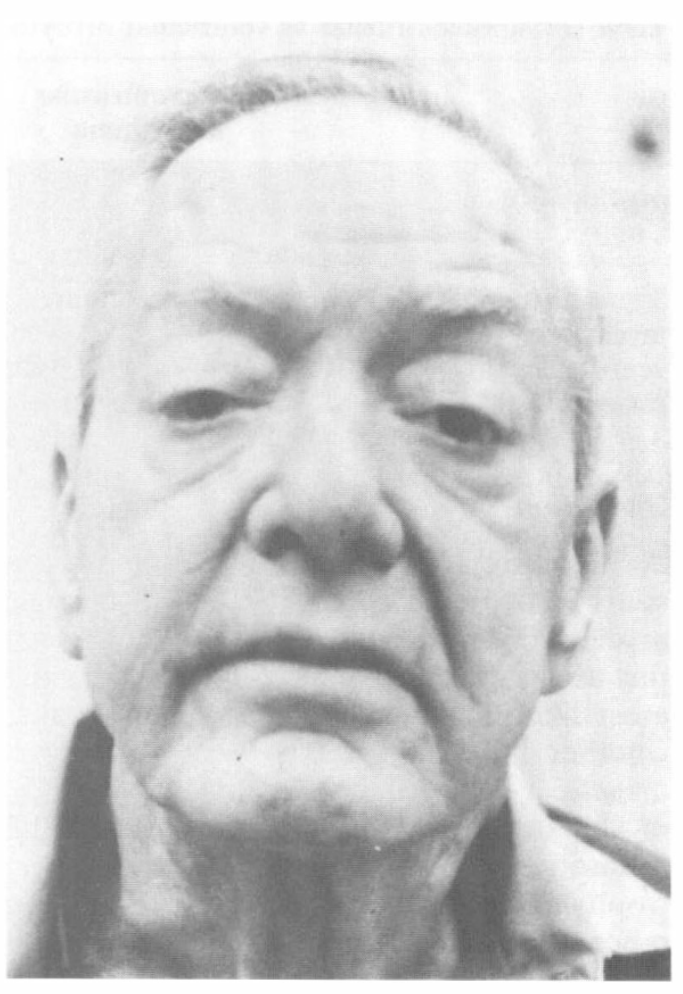

Figure 2 Frontal view of a subject with $27 \mathrm{~mm}$ exophthalmos.

Table I Eye signs in patients with ocular protrusion by exophthalmometry

\begin{tabular}{ll}
$\begin{array}{l}\text { Convergence weakness and lid lag, } \\
\quad \text { unilateral }\end{array}$ & 1 \\
Convergence weakness, unilateral & 1 \\
Lid lag & 2 \\
Diplopia with fatigue & 1 \\
Nystagmus, unilateral & 1 \\
No findings & 2 \\
Total & 8 \\
\hline
\end{tabular}

thyroxine (T4) and triiodothyronine (T3) levels but non suppression of thyroid stimulating hormone (TSH) and low levels of T3 resin uptake. Thyroxine binding protein was elevated in both patients. The interpretation was hyperthyroxinemia due to increased serum binding of thyroid hormone. ${ }^{10}$ Neither patient had complaints of nervousness, fatigue, palpitation, weight loss, or change in bowel habits to suggest hyperthyroidism. 
Table II Supraventricular vs ventricular arrhythmias: myelopathy vs control subjects

\begin{tabular}{lccc}
\hline & $\begin{array}{c}\text { Supraventricular } \\
\text { arrhythmia }\end{array}$ & $\begin{array}{c}\text { Ventricular } \\
\text { arrhythmia }\end{array}$ & $\begin{array}{c}\text { Total subjects } \\
\text { with arrhythmia }\end{array}$ \\
\hline $\begin{array}{l}\text { Myelopathy } \\
\text { Control }\end{array}$ & 25 & 9 & 34 \\
& 11 & 15 & 26
\end{tabular}

Supraventricular arrhythmia was predominately PAC in $15, \mathrm{AF}$ in 9 , and AT in 1 in the myelopathy group, PAC in $5, \mathrm{AF}$ in 6 in the control group. The ratio of supraventricular to ventricular arrhythmia was significantly greater in the myelopathy group: 2.8 for myelopathy patients vs 0.7 for control subjects. Chi square $=5.9842, p<0.02$.

\section{Discussion}

A high prevalence of exophthalmos was confirmed, being found in $12 \%$ of chronic myelopathy patients, previous reports of this association not being found. By far the most likely explanation for exophthalmos, whether bilateral or unilateral, is Graves' disease. ${ }^{11}$ Computerized tomography (CT) of the orbits was carried out in 2 of the proptotic subjects and characteristic hypertrophy of extraocular muscle was found. No other explanations for ocular protrusion such as tumor cysts, granuloma, or arteriovenous fistula were found. The other 7 patients did not receive CT scanning.

Although the onset of exophthalmos is closely related to the onset of hyperthyroidism, the persistence of exophthalmos is observed in the euthyroid state, after the remission of hyperthyroidism. ${ }^{1,2}$ The possibility of having overlooked earlier episodes of thyrotoxicosis exists because many symptoms are mediated through the sympathetic nervous system, which, in the myelopathy patient is badly damaged. ${ }^{12}$ Thus the palpitation, tremor, restlessness, and weakness, characteristic of thyrotoxicosis and controlled with sympatholytic agents in the neurologically intact man, ${ }^{13}$ might be less dramatic in the spinal man. For example, 2 patients in another group of myelopathy patients followed at the nearby Veterans Administration hospital at West Roxbury developed acute proptosis. One was proven to have thyrotoxicosis, but one was not tested at the onset of exophthalmos.

A high fraction of all arrhythmias in the myelopathy patients were supraventricular as compared with controls, even though the groups were matched for age, which is a major determinant of the common arrhythmias-premature atrial contraction, atrial fibrillation, and premature ventricular contraction. ${ }^{14,15}$ Stress, fatigue, smoking, alcohol abuse, caffeine intake, use of cardiac glycosides, and underlying heart disease were not compared in the groups surveyed, but these factors induce both supraventricular and ventricular arrhythmias. ${ }^{4}$

References cite supraventricular arrhythmias in hyperthyroid patients in perhaps $20 \%$, and note that atrial fibrillation persists after the onset of euthyroidism in half of these cases. ${ }^{3,16}$ It is conceivable that residual cardiac as well as ocular effects are being observed. No other reports of these eye and heart abnormalities in myelopathy patients have been found.

The high prevalence of hypothyroidism in myelopathy patients has been reported, however. Prakash et al found a $0.9 \%$ prevalence as compared with the $2.7 \%$ prevalence reported here, both rates being some 10 times greater than that reported for men in a general population, $<0.1 \% .{ }^{17}$ If it is recalled that over many years the eventual course of thyrotoxicoses, even when untreated by surgery or irradiation of the thyroid gland, is one of hypothyroidism, ${ }^{4}$ then the possibility of a residuum of Graves' disease in the myelopathy patients can be raised again.

Taken in separate groups, the 9 patients with exophthalmos, the 25 with supraventricular arrhythmia, and the 2 with hypothyroidism can suggest a disposition of the myelopathy patient to Graves' disease. Taking only patients with a combination of conditions, there are 3 , these having both proptosis (extensive) and atrial fibrillation. 
Even this smaller number of suspected Graves' disease is, at a $3.9 \%$ prevalence, much greater than expected, $0.2 \% .^{18}$

Graves' disease is considered an autoimmune disorder with genetic predisposition. ${ }^{19}$ An immune reaction to a bacterial infection has been implicated, however, in Scandinavian populations with enteropathies due to Yersinia enterocolitica. ${ }^{20}$ The considerable bacterial infection of the urinary tract and the large bowel stasis with implicit bacterial overgrowth in the myelopathy patient may be relevant to this problem.

In conclusion it is suggested that acute and chronic myelopathy patients be observed for the onset of Graves' disease. The early detection of exophthalmos permits a more effective treatment, with steroids and radiation, ${ }^{21}$ of this sometimes disfiguring complication of myelopathy. The anticoagulation of the patient with the onset of atrial fibrillation can prevent an associated systemic thromboembolic event. ${ }^{3,22}$

\section{Acknowledgment}

Dr Ranji N Samaraweera was helpful in the interpretation of the computerized tomograms in this study.

\section{References}

1 Gorman CA (1983) Temporal relationship between onset of Graves' ophthalmopathy and diagnosis of thyrotoxicosis. Mayo Clin Proc 58: 515-9.

2 Jones IS (1962) The orbit: a review of the literature for 1961. Arch Ophthalmol 68: 399-426.

3 Staffurth JS, Gibberd MC, Tang-Fui SN (1977) Arterial embolism in thyrotoxicosis with atrial fibrillation. Br Med J 2: 688-90.

4 Wood LC, Ingbar SH (1979) Hypothyroidism as a late sequela in patients with Graves' treated with antithyroid agents. J Clin Invest 64: 1429-36.

5 Musch DC, Frueh BR, Landis JR (1985) The reliability of Hertel exophthalmometry. Ophthalmology 92: 1177-80.

6 Frueh BR, Garber F, Grill R, Musch DC (1985) Positional effects on exophthalmometer readings in Graves' eye disease. Arch Ophthalmol 103: 1355-6.

7 Migliori ME, Gladstone GJ (1984) Determination of the normal range of exophthalmometric values for black and white adults. Am J Ophthalmol 98: 438-42.

8 Marriott HJL, Myerburg RJ (1990) Recognition of cardiac arrhythmias and conduction disturbances. In: Hurst JW, Schlant RC, editors: The Heart. McGraw Hill Inc: 489-534.

9 Kaplan MM, Larsen PR, Crantz FR, Dzan VJ, Rossing TH, Haddow JE (1982) Prevalence of abnormal thyroid function tests results in patients with acute medical illness, Am J Med 72: 9-15.

10 Ikekubo K, Konishi J, Endo K, Nakajima K, Okuno T, Kasagik K (1982) Anti-thyroxine and anti-triiodothyronine antibodies in three cases of Hashimoto's thyroiditis. Acta Endocrinol 89: 557-66.

11 Trokel SL, Jakobiec FA (1981) Correlation of CT scanning and pathologic features of Graves' disease. Ophthalmology (Rochester) 88: 553-64.

12 Corbett JL, Frankel HL, Harris PJ (1971) Cardiovascular responses to tilting tetraplegic man. J Physiol 215: 411-31.

13 Dillon PT, Babe J, Meloni CR, Canary JJ (1970) Reserpine in thyrotoxic crisis. N Engl J Med 283: 1030-1033.

14 Hiss RG, Lamb LE (1962) Electrocardiographic findings in 122,043 individuals. Circulation 25: 947-61.

15 Kannell WB, Abbott RD, Savage DD, McNamara PM (1982) Epidemiological features of chronic atrial fibrillation. New Engl J Med 306: 1018-22.

16 Ladenson PW (1990) Recognition and management of cardiovascular disease related to thyroid dysfunction. Am J Med 88: 638-41.

17 Prakash V, Lin MS. Song CH, Perkash I (1980) Thyroid hypofunction in spinal cord injury patients. Paraplegia 18: 56-63.

18 Tunbridge WMG, Evered DC, Hall R, Appleton D, Brewis M, Clark F et al (1977) The spectrum of thyroid disease in a community: the Whickham Survey. Clin Endocrinol 7: 481-93.

19 Doniach D (1975) Humeral and genetic aspects of thyroid autoimmunity. Clin Endocrinol Metab 4: 267-285.

20 Shenkman L, Bottone EJ (1976) Antibodies to Yersinia entero-colitica in thyroid disease. Ann Intern Med 85: 735-9.

21 Donaldson SS, Bagshaw MA, Kriss JP (1973) Supervoltage orbital radiotherapy for Graves' ophthalmopathy. J Clin Endocrinol Metab 37: 276-85.

22 Braunwald E (1980) Valvular heart disease. In: Braunwald E, editor. Heart Disease. WB. Saunders Company, Philadelphia: 1105. p. 